\title{
Stochastic Resonance Control by Coupling Two Fractional Bistable Systems
}

\author{
Yongjun Zheng ${ }^{1,2}$, Fei Wang ${ }^{2}$ and Shan-an Zhu ${ }^{1}$ \\ ${ }^{1}$ College of Electrical Engineering, Zhejiang University, Hangzhou, Zhejiang 310027, \\ China \\ ${ }^{2}$ College of Metrology and Measurement Engineering, China Jiliang University, \\ Hangzhou, Zhejiang 310018, China \\ davidzhyj@cjlu.edu.cn,316225232@qq.com,zsa@zju.edu.cn
}

\begin{abstract}
In order to enhance or produce stochastic resonance (SR), to obtain the required output response with specific properties, two bistable systems based on fractional over-damped Langevin equation are coupled into one multi-steady-state system via nonlinear method, one fractional bistable system where inherent parameters can be controlled is fed into the other system, the kinetic characteristics of the new coupled system is found much richer than each single fractional system. Theoretical analysis and numerical simulation results show that the $S R$ can be enhanced or produced by adjusting parameters as coupling coefficient, fractional system inherent parameters as well as the external periodical signal amplitude while another fractional system's parameters fixed. The coupled system has high practical value, which provides a reliable theoretical basis for the control and its applications of artificially generated stochastic resonance.
\end{abstract}

Keywords: stochastic resonance, coupled fractional bistable system, control, fractional over-damped Langevin equation

\section{Introduction}

Benzi et al., put forward the concept of stochastic resonance in 1981, in the process of analyzing the signal, the noise is generally considered as harmful signal, signal-to-noise ratio(SNR) reduced because of its presence, which has an impact for extracting useful information, but in some special nonlinear system, the noise strengthen the ability to detect of weak signal [1]. During the past 30 years, many researchers have done more in-depth study for stochastic resonance. Now stochastic resonance has been widely applied to various industries, for example, signal detection in the marine noise environment [2], improving detection capability of neurons for weak tactile signal [3], detecting of chemical weak signal [4] and so on.

But most stochastic resonance studies are based on integer order calculus, which has localized characteristic and cannot well consistent with experimental results. In recent years, fractional calculus are widely used in fluid mechanics, image processing, seismic analysis, fractional PID controllers [5-8], compared to the integer order, it has global relevance characteristic, and can well describe the history-dependent system functions process. For fractional order system fewer parameters are required but can be obtained very good results.

Stochastic resonance described by fractional calculus was interested by many researchers recently. Stochastic resonance phenomenon was appeared when applying sufficiently high noise intensity to a bistable duffing potential system with fractional derivative order, the critical value of noise level changes by the derivative order. The author found that when 
derivative order $\mathrm{q}$ is larger than one certain value, the single hop between potential wells is not occurred [9]. The authors investigated the stochastic resonance phenomenon described by underdamped linear fractional Langevin equation, the results show that both the output amplitude and SNR reach a maximum with the changing of driving frequency, which indicates that the SR phenomenon appears [10]. A fractional bistable system where the fractional calculus is defined by Caputo method is used to detect weak signals, SR phenomenon is occurred, the spectral power amplification (SPA) of input and output signals has a non-monotonic function with noise intensity, fractional order and bistable system parameters. It is also founded that when increasing the fractional order q the SPA decrease sharply, which indicates that the SR phenomenon vanishes [11]. So it is meaningful to discuss and investigate the generation and enhancement of SR under the circumstances that SR is not occurred mentioned in the above papers.

Gammaitoni et al., first proposed the method to control stochastic resonance, in order to suppress or enhance the spectrum value of the output power spectrum at the input signal frequency [12]. After that, a variety of different stochastic resonance methods have been proposed in different systems [13-14], which promotes the application of stochastic resonance. Stochastic resonance can be occurred when synergistic effect of three elements, they are weak periodic signal, noise signal and nonlinear systems. For the fractional overdamped Langevin equation, SR is generated when the parameters achieved synchronization, which are weak signal's amplitude and frequency, the intensity of the noise signal and the structural parameters of nonlinear systems [15]. Changing these parameters can make fractional systems stochastic resonance phenomenon, but in the actual production and applications, weak periodic signal and the noise signal, and nonlinear structural parameters tend to be constant, changing its barrier height is not possible. Therefore, in order to make the output signal generate or enhance stochastic resonance, according to response theory of coupled bistable system [16], in this paper, two fractional biatable systems are coupled into a multi-stable system, we analyze the response of the coupled system under weak periodic signal and the noise signal, as well as the effects of the coupled coefficient, the controlsystem parameters and external periodic signal on stochastic resonance.

The paper is organized as follows. In section II, we introduce the models and methods of single fractional bistable system based on over-damped fractional Langevin equation. SR occurs when the fractional order is less than one certain value, if it is greater than the threshold there is no SR. In section III, coupled models with two fractional bistable systems are introduced and analyzed. Section IV shows the numerical results with one parameter variable while others fixed. Section V is some discussions and conclusions.

\section{Single Fractional Bistable System Model}

The single fractional bistable system is based on an over-damped fractional Langevin equation $[11,15]$ :

$$
{ }_{0}^{c} D^{\alpha} x(t)=a_{0} x-b_{0} x^{3}+A \cos (2 \pi f t)+\xi(t)
$$

where ${ }_{0}^{c} D^{\alpha} x(t)$ is the $\alpha$ order fractional order derivative to $x(t)$ by using Caputo's definition, and $0<\alpha<1$, the Caputo's definition is written as

$$
{ }_{a} D_{t}^{\alpha} f(t)=\frac{1}{\Gamma(n-\alpha)} \int_{a}^{t} \frac{f^{(n)}(\tau)}{(t-\tau)^{\alpha-n+1}} d \tau
$$


We take a lower limit $a=0$ for the above definitions. $A \cos (2 \pi \omega t)$ is an external signal with amplitude $A$ and frequency $\omega$, and $\xi(t)$ is a zero-mean Gaussian white noise, whose autocorrelation function is $\langle\xi(t) \xi(0)\rangle=2 D \delta(t), D$ is the intensity of noise.

$a_{0} x-b_{0} x^{3}$ is a bistable potential with two minima located at $\pm x_{m}$, they are separated by a potential barrier with height $\Delta V=a^{2} /(4 b)$. When the external periodic forcing is too weak, the particle cannot jump periodically from one potential well into the other. It can be seen from Eq. 2 the Caputo's definition of fractional order differential that the fractional order $p$ relates with the memory characteristic, the larger $p$ means the memory characteristic much less, when $p \rightarrow 1$, it turns into the integer differential order, which indicates totally loss memory, while $p \rightarrow 0$, it differential equal to constant 1, that indicates the same memory characteristic to the speed of each time. When there is no noise $\xi(t)$ applied to the Eq.1, just applying the periodic driving force $A \cos (2 \pi \omega t)$, the output responses are observed as the fractional order changed. Figure 1 indicates the curves when fractional order $p$ changes from 0.9 to 0.1 with step 0.1 , the other parameters are $a_{0}=b_{0}=1, A=0.3, f=0.01$. From Figure 1 we can see when the fractional order $\alpha$ attenuated from 1 to 0 , the particle is doing partial periodic motion around the balance point $x=1$ or $x=-1$ with frequency $f=0.01$, when $\alpha$ reaches a threshold $\alpha_{t}=0.29$, the particles hop the potential barrier top which takes place at $x=0$ into the other well, thus do the periodical motion between $x= \pm 1$ with the center at $x=0$. When $\alpha$ is smaller than $\alpha_{t}$, the particles can hop the potential barrier top without external noise energy, thus the SR phenomenon occur. While $\alpha$ is greater than $\alpha_{t}$, the particles just do partial periodic motion around one well, it only needs the external noise synchronization to produce SR phenomenon.

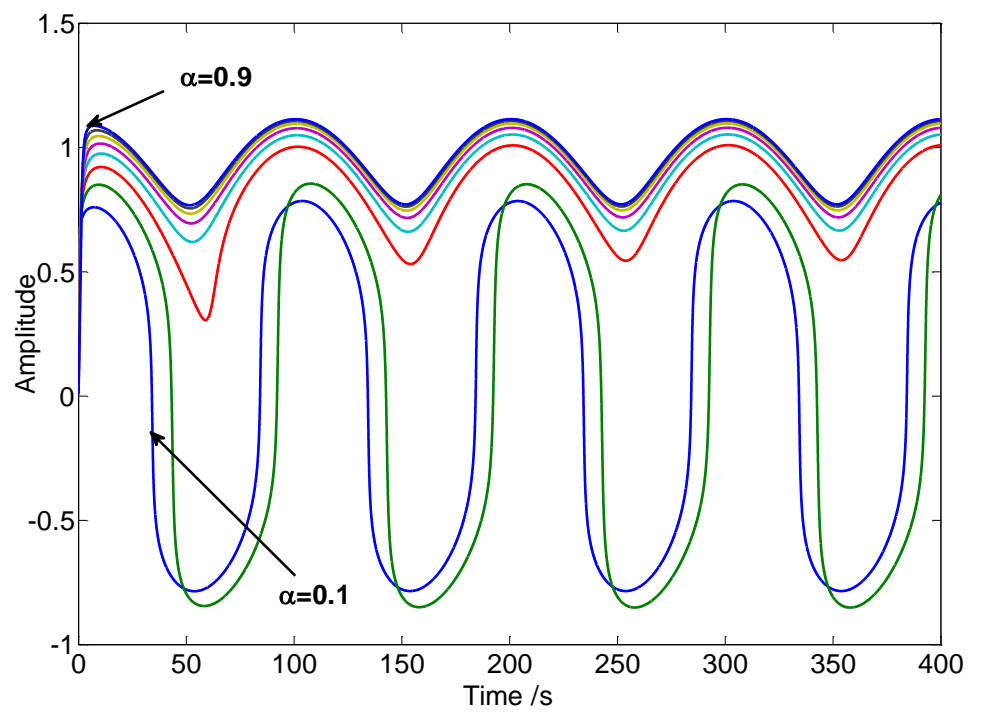

Figure 1. Output Response with Different Fractional Order $\alpha$

When applied the external noise but its intensity is not matched well, the energy of output signal $x(t)$ is not strong enough to make the particle jump over the barrier top into another well, the SR will not be occurred. It can be seen from Figure 2(a), where the external noise $\xi(t)$ is applied with intensity $D=2$, keeping the other parameters unchanged. The three 
elements as periodic signal, noise signal and fractional system itself are not synergistic, there is no SR phenomenon, and the power spectrum of output signal is very small, the value is 0.08884, as shown in Figure 2(b).
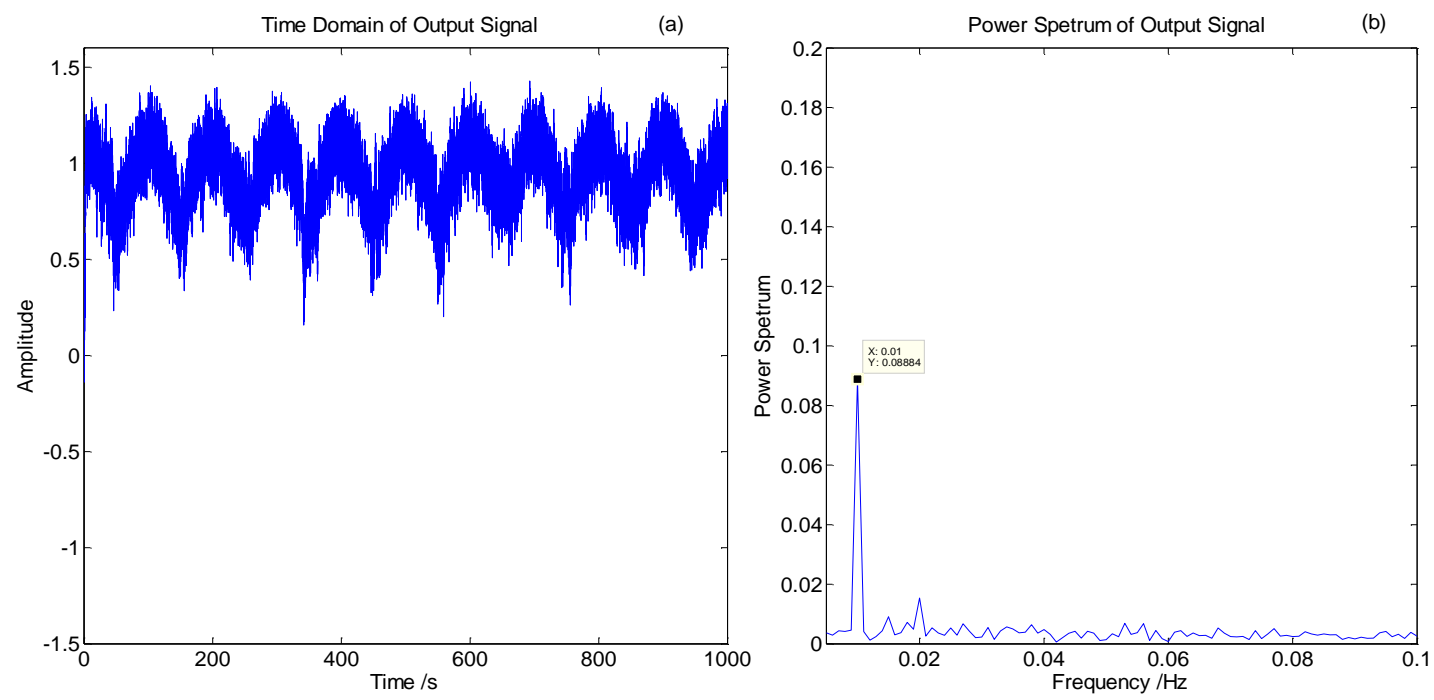

Figure 2. Time Domain and Power Spectrum of Output Signal

\section{Fractional Coupled Models}

Concerned the situation that the actual variables of fractional systems tend not to be matched, there is no stochastic resonance phenomenon, in order to artificially control the system to produce or enhance stochastic resonance phenomenon, we constitute two fractional systems with nonlinear coupling method into one coupled fractional system, one fractional system whose bistable potential equation is $\left(a_{0} x-b_{0} x^{3}\right)$ with its parameters fixed is taken as a controlled system, another fractional system with $\left(a y-y^{3}\right)$ whose parameters are adjustable as a controllable system, and additional periodic signal is introduced into the system, the block diagram of coupled fractional system is shown as Figure 3.

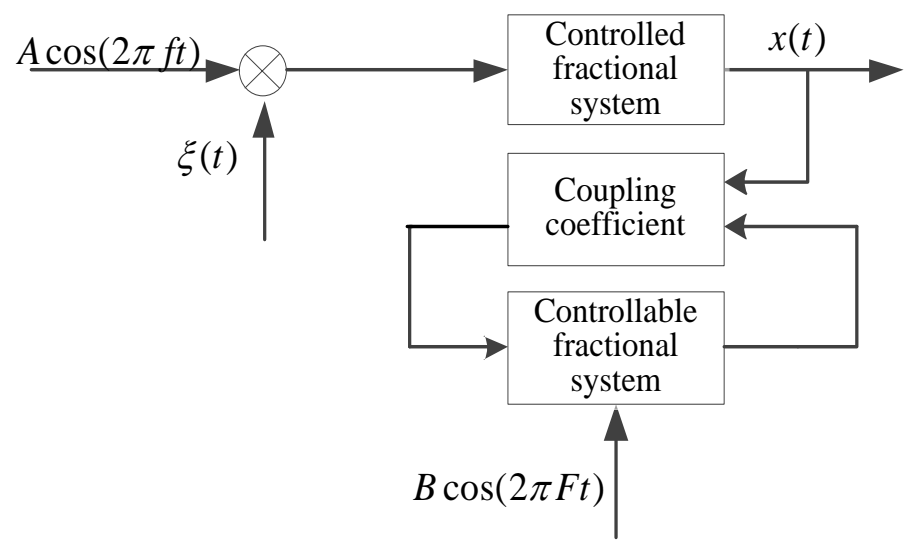

Figure 3. Block Diagram of the Coupled Fractional System 
In Figure 3 the relations of variables and parameters of coupled fractional system are expressed by the following two fractional Langevin equations:

$$
\begin{aligned}
& D^{\alpha} x(t)=a_{0} x-b_{0} x^{3}+A \cos (2 \pi f t)+\xi(t)-\gamma x y^{2} \\
& D^{\alpha} y(t)=a y-y^{3}+B \cos (2 \pi F t)-\gamma x^{2} y
\end{aligned}
$$

where $\gamma$ is the coupled coefficient between the two fractional systems, $a_{0}$ and $b_{0}$ are the parameters of the controlled fractional system. $A \cos (2 \pi f t)$ and $\xi(t)$ are the weak periodic signal and the noise signal of controlled fractional system, the above parameters are fixed. $a$ is the structural parameter of variable fractional system, $B \cos (2 \pi F t)$ is the applied periodic signal of the control system, by changing the structural parameter $a$ of the system, coupled coefficient $\gamma$, external periodic signal amplitude $B$, the stochastic resonance can be occurred or enhanced.

\section{Numerical Simulation Analyses}

Based on the block diagram of fractional coupled control system, we adopt the signal fractional system mentioned in Section 2, the parameters of controlled fractional system are as follows: $a_{0}=b_{0}=1$, weak periodic signal's amplitude $A=0.3$, frequency $f=0.01$, fractional order $\alpha=0.75$, and the noise signal strength $D=2$. The stochastic resonance phenomenon is not appeared when there is no coupling effect. We try to adjust the controllable fractional system, one parameter is changed while the others are fixed, the time response and power spectrum of output signal are observed and analyzed.

\section{1. $B, F$ and $a$ Fixed, Changing $\gamma$}

The amplitude $B=1$ and frequency $F=11$ of coupled control system's input periodic signal, and the structural parameter $a=1.25$ of control system are fixed, we change the coupled coefficient $\gamma$. When the coupled coefficient $\gamma=0.03$, the time domain output response is shown as Figure 4(a). The barrier top of coupled system is high enough that the particles cannot jump from one potential well into other one, the output signal is fluctuate around one potential well. This is because the coupling coefficient is too small, there is no enough energy being coupled into the controlled system, which let the weak periodic signal has not enough energy to cross the barrier top, thus the stochastic resonance does not be occurred, so that the power spectrum of the output signal $x(t)$ at frequency $f=0.01$ is very small as 0.09704 , as shown in Figure 4(b). 

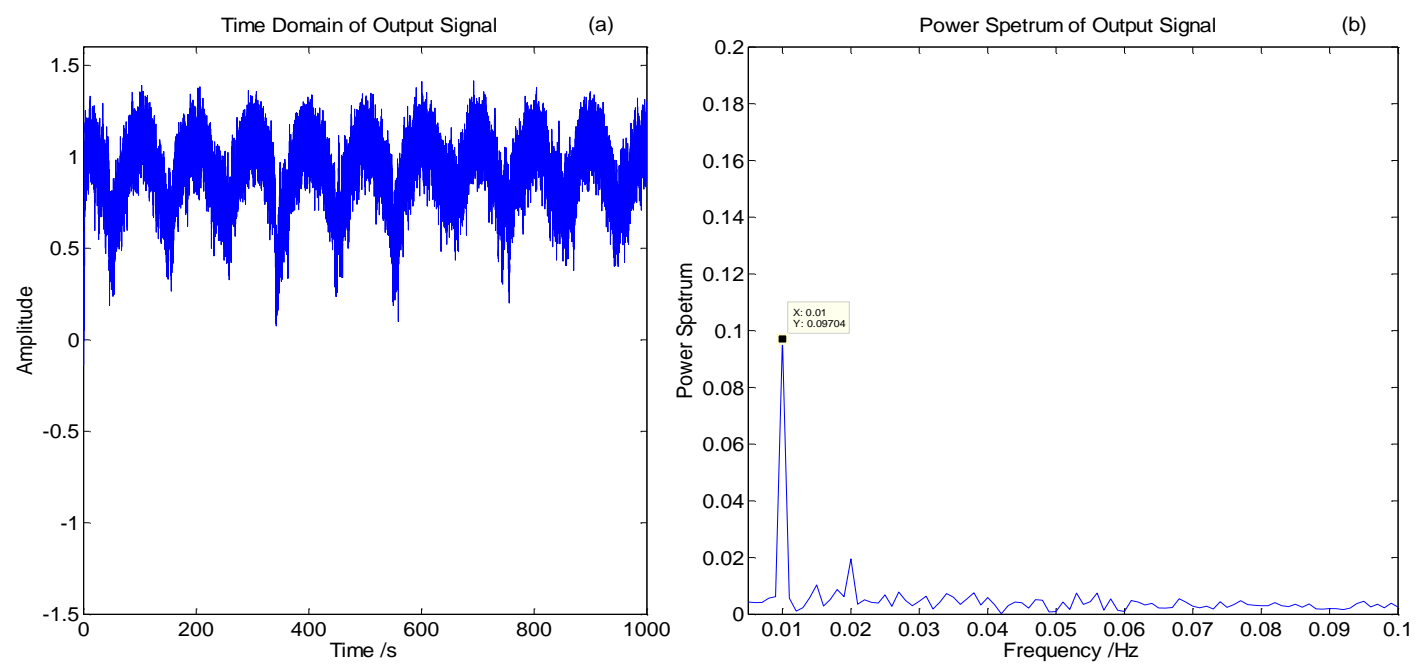

Figure 4. Time Domain and Power Spectrum of Output Signal when $\gamma=0.1$

While continuing to increase the coupling coefficient to 0.3 , the output signal of coupled system can cross the barrier top and fluctuate back and forth in two stable potential wells as shown in Figure 5(a). The reason is that when the coupling coefficient increases, more energy from the controllable system was coupled into the controlled system, making more energy being transferred and provide the energy to the weak periodic signal, let it possible to cross the barrier top, and can fluctuate back and forth in the two potential wells, the synergistic effect was produced between the two coupling systems, thus the stochastic resonance phenomenon is appeared. It can be seen from Figure 5(b), the power spectrum of the output response $x(t)$ at frequency $f=0.01$ is 0.503 , so that the energy of output signal is mainly focused near frequency $f=0.01$, thus it can improve the quality of the output response $x(t)$, which has a very important value for the practical application.
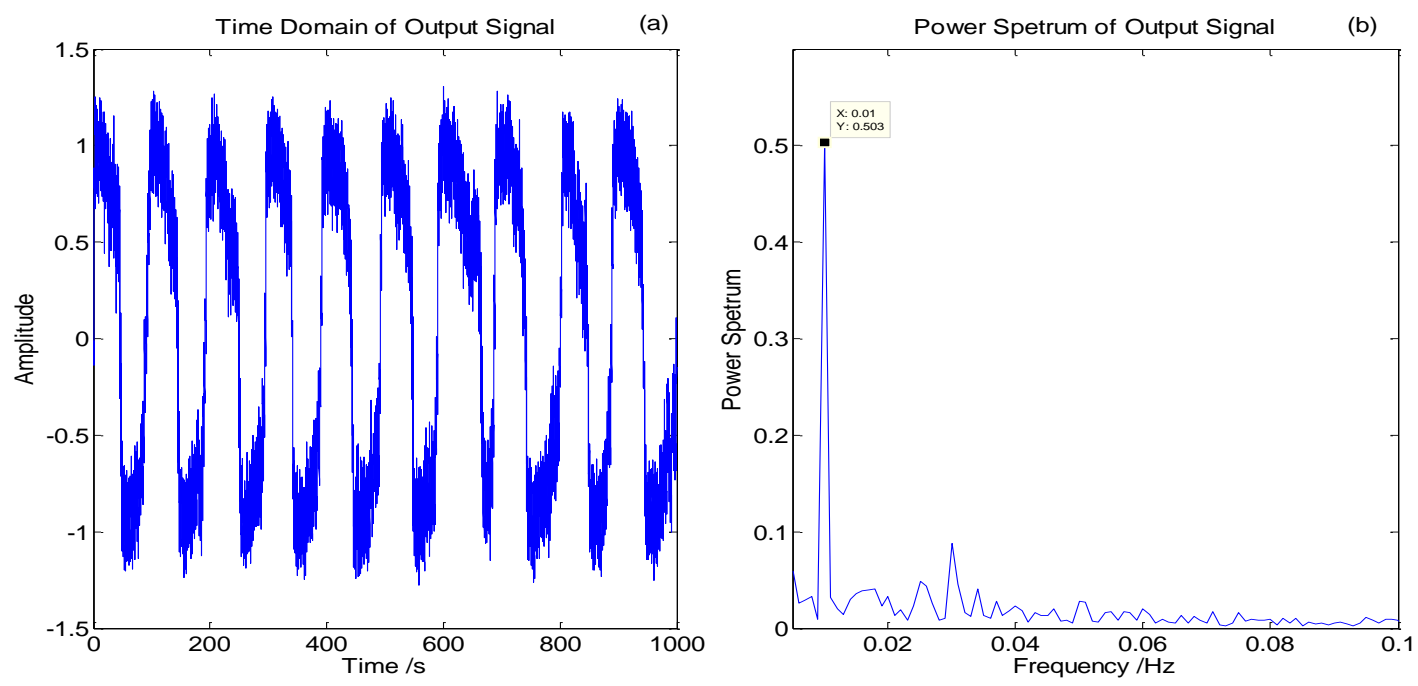

Figure 5. Time Ddomain and Power Spectrum of Output Signal when $\gamma=0.3$ 
When the coupled coefficient $\gamma$ is increased to 2, which means there is much more energy exchange between controlled and controllable system, the energy to cross the barrier top of controlled system increases much more than the energy to jump over it, so the energy transform between noise signal and weak periodic signal has no effect, so that the output in response to the value of the power spectrum in the frequency becomes small, it can be seen from Figure 6(a), there is no stochastic resonance occurs, the power spectrum of the output response $x(t)$ at frequency $f=0.01$ becomes small as 0.08905, as shown in Figure 6(b).
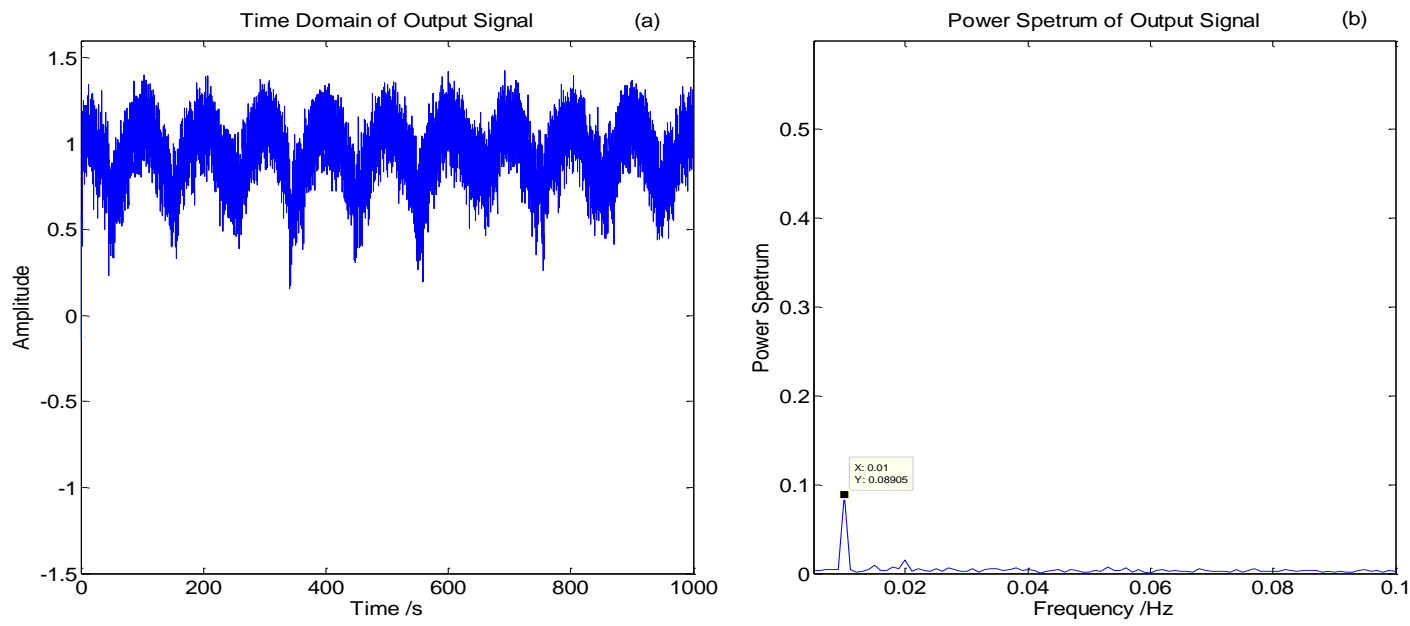

Figure 6. Time Domain and Power Spectrum of Output Signal when $\gamma=2$

It can be drawn from the above three figures, the introduction of coupled fractional control system, by increasing its coupled coefficient $\gamma$, which can enhance stochastic resonance of the coupled controlled system, but continue to increase the structural parameter value, then stochastic resonance of coupled controlled system will be restrained. When the other conditions are certain, as shown in Figure 7, the relationship between the power spectrums at fixed frequency with structural parameters of coupled fractional control system is nonmonotonic, there is one value $\gamma$ makes the power spectrum at fixed frequency maximum, and optimal stochastic resonance phenomenon is presented.

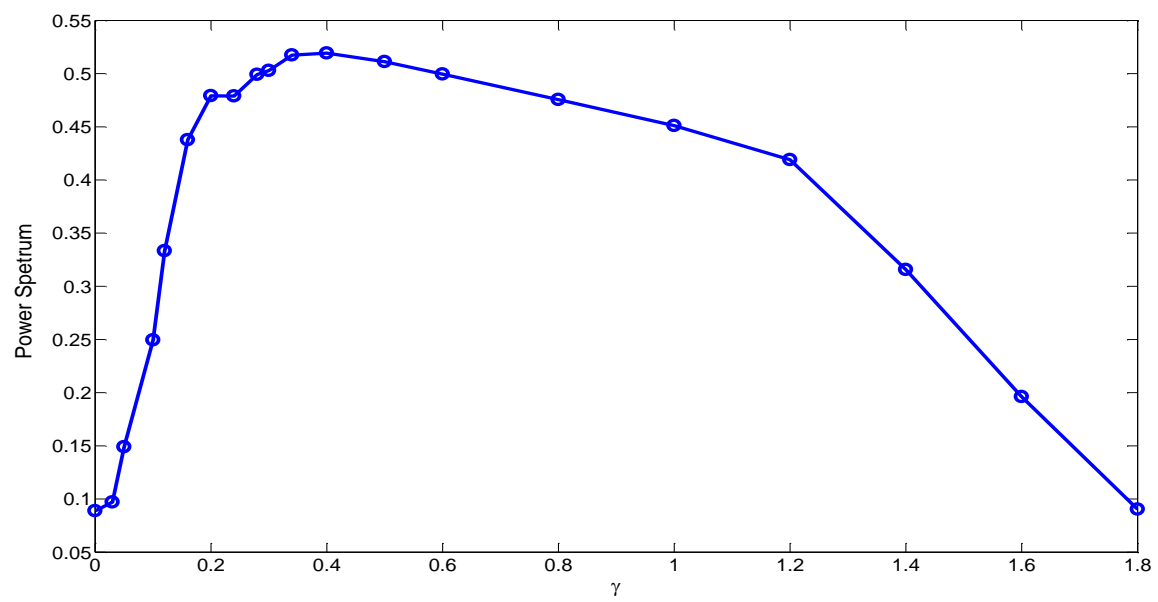

Figure 7. The Relation between $\gamma$ and the Power Spectrum at $f=0.01$ 


\section{2. $B, F$ and $\gamma$ Fixed, Changing $a$}

When the amplitude $B=1$ and frequency $F=11$ of coupled control system's input periodic signal, and the coupled coefficient $\gamma=0.3$ are fixed, by changing the structural parameter $a$, the relation between the power spectrum at $f=0.01$ and $a$ is shown as Figure 8(a).

The other parameters kept consistent indicates the signal energy of controlled factional system without change, changing structural parameter $a$ will couple and transmit the energy of controllable fractional system into the controlled one, make the particle in bistable potential well has more energy to cross over the barrier height, the barrier height is same because the controlled system's parameters are consistent, thus the synergistic action between the noise signal and output signal happened, the jumping frequency between two potential wells is synchronous with the weak signal's frequency, making the three elements as weak signal, noise signal and controlled fractional system become to synergistic, thus the SR phenomenon occurs. When the structural parameter $a$ is small, the coupling effect is not obvious, when it is increased, the coupling effect and energy transmission is enhanced and the power spectrum at weak signal's frequency $f=0.01$ is increasing, when it is adjusted into 1.5, the SR phenomenon is most obvious, and the power spectrum is 0.5113 , much larger than its original one 0.0724 when applied no coupling fractional system. The time domain and power spectrum of output signal at $a=1.5$ is shown as Figure 9. But when the structural parameter $a$ is increased much more such as $a=6.0$, which means there is much more energy exchange between controlled and controllable system, the energy to cross the barrier top of controlled system increases much more than the energy to jump over it, so the energy transform between noise signal and weak periodic signal has no effect, so that the output in response to the value of the power spectrum in the frequency becomes small, there is no stochastic resonance occurs, the power spectrum of the output response at frequency becomes small as 0.156.
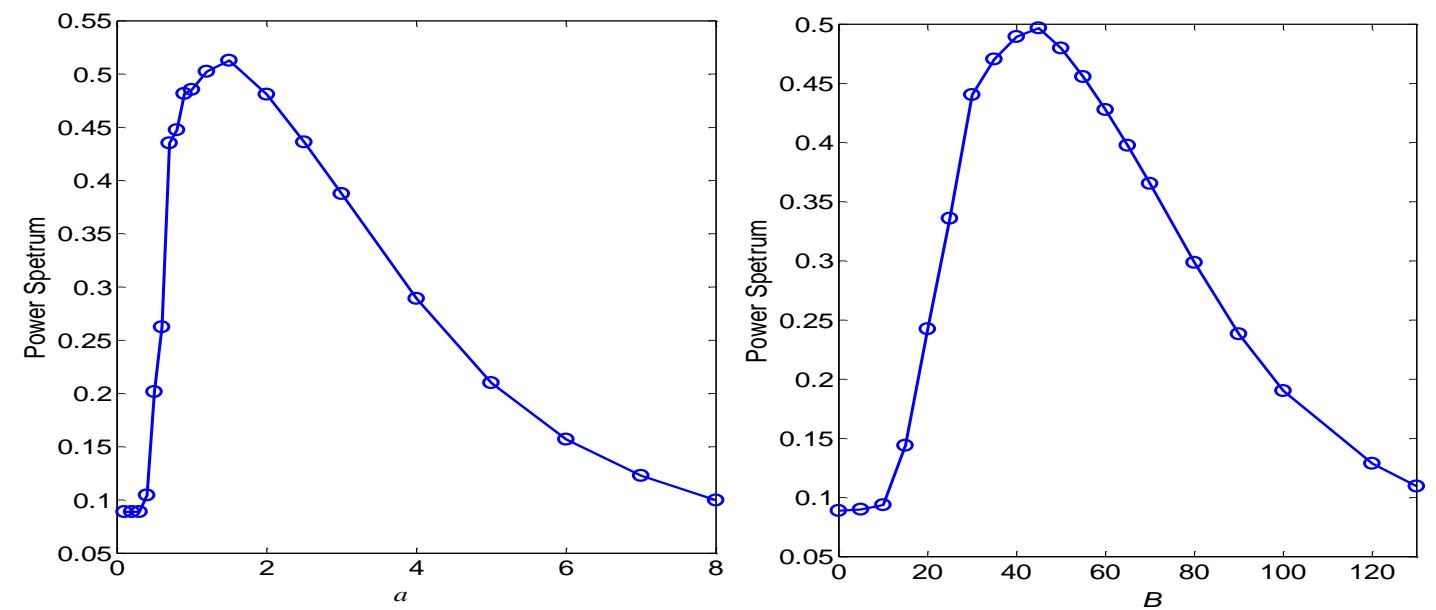

Figure 8. (a) Power Spectrum versus $a$ at $f=0.01$ (b) Power Spectrum versus $B$ at $f=0.01$ 

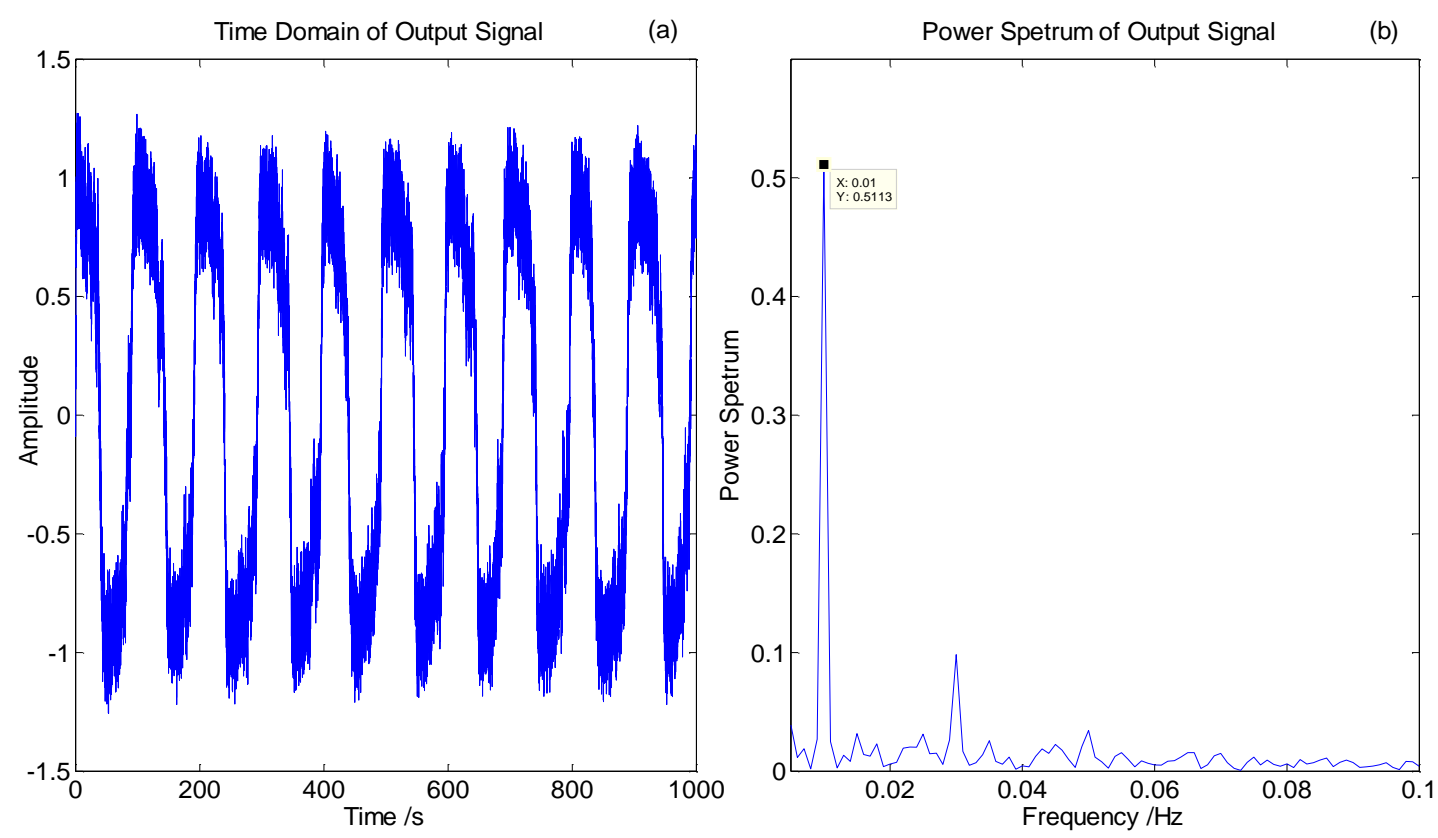

Figure 9. Time Domain and Power Spectrum of Output Signal at $a=1.5$

\section{2. $F, \gamma$ and $a$ Fixed, Changing $B$}

When $F=11, \gamma=0.3, a=0.25$ are fixed, the relation between the power spectrum at $f=0.01$ and amplitude $B$ is shown as Figure 8(b).

When the periodic signal's amplitude $B$ is small, the coupling effect is not obvious, when it is increased, the coupling effect and energy transmission is enhanced and the power spectrum at weak signal's frequency $f=0.01$ is increasing, when it is adjusted into 45, the SR phenomenon is most obvious, and the power spectrum is 0.4949 , much larger than its original one 0.0884 when applied no coupling fractional system. The time domain and power spectrum of output signal at $B=45$ is shown as Figure 10. That indicates the synergistic action between the noise signal and output signal happened, the jumping frequency between two potential wells is synchronous with the weak signal's frequency, making the three elements as weak signal, noise signal and controlled fractional system become to synergistic, thus the SR phenomenon occurs.

But when the periodic signal's amplitude $B$ is increased much more such as $B=120$, which means there is much more energy exchange between controlled and controllable system, the energy to cross the barrier top of controlled system increases much more than the energy to jump over it, so the energy transform between noise signal and weak periodic signal has no effect, so that the output in response to the value of the power spectrum in the frequency becomes small, there is no stochastic resonance occurs, the power spectrum of the output response at frequency becomes small as 0.1287 . 

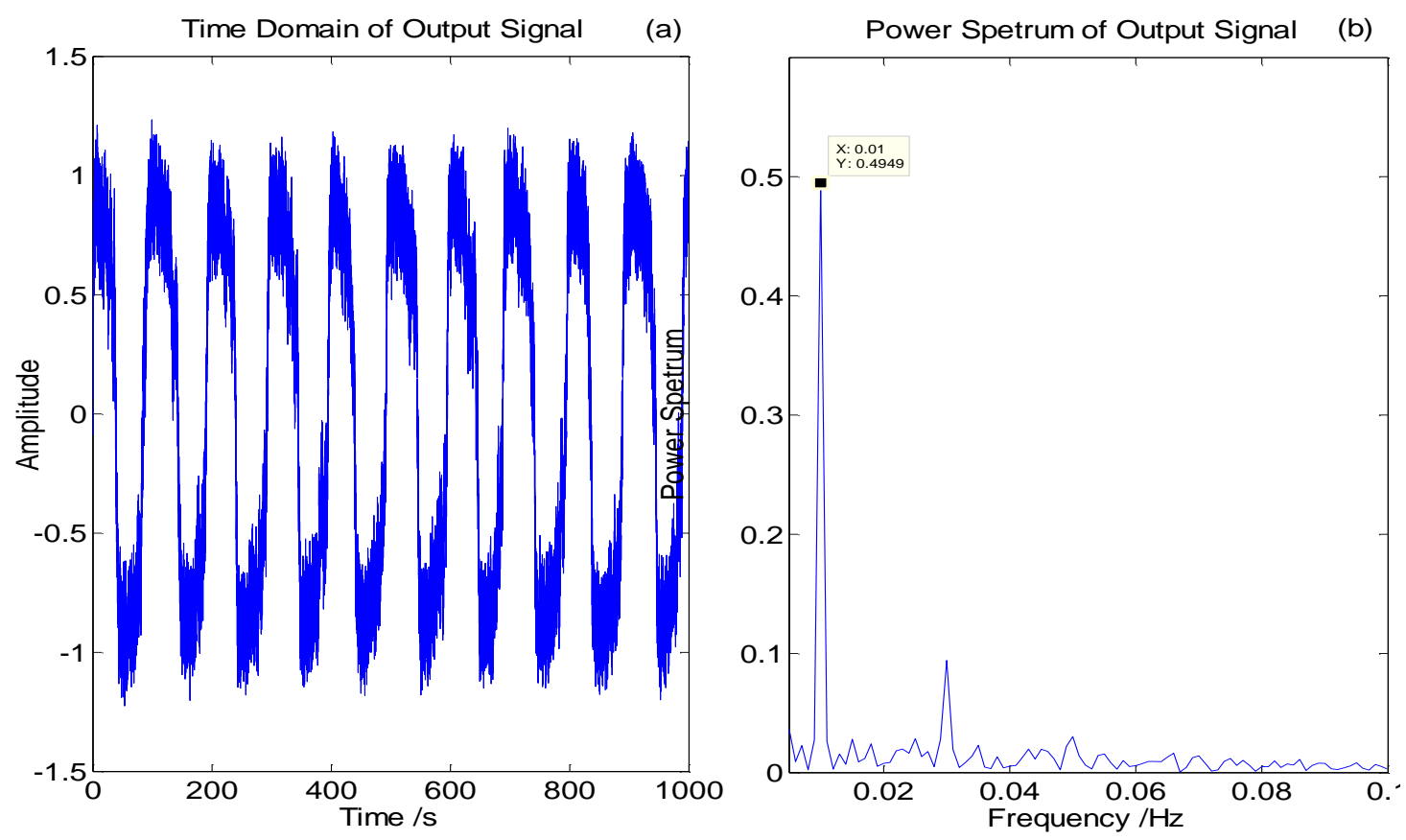

Figure 10. Time Domain and Power Spectrum of Output Signal at B=45

From Figure 7 and Figure 8, it can be concluded that the power spectrum at the weak periodic signal's frequency of controlled fractional system has non-monotonic relation with structural parameter $a$, coupling coefficient $\gamma$ or applying external periodic signal amplitude $B$, when the other parameters kept unchanged, the power spectrum first increase then decrease, there is a maxima, that presents the optimal SR phenomenon.

\section{Conclusions}

This paper mainly introduces the concept of coupling system, one fractional system with parameters fixed as the controlled system, and then another with parameters variable as a controllable system, through a certain nonlinear relationship to make two systems coupled, then research the time domain and frequency characteristics of controlled fractional-system output response. We proposed a method to control stochastic resonance, by analyzing the barrier changing or energy conversion of the coupled fractional controlled system, combine with changes of its output response to determine the relationship between these variables and the state of stochastic resonance of the system, to control stochastic resonance of fractional system. We found the non- monotonic relation between the power spectrum and each adjustable parameter, by adjusting each parameter as structural parameter, coupling coefficient or external periodic signal amplitude while the others unchanged, the SR phenomenon is controllable to be produced or enhance.

\section{Acknowledgments}

This project is supported by the National Nature Science Foundation of China, Grant No. 50675214. 


\section{References}

[1] R. Benzi, A. Sutera and A. Vulpiani, "Journal of Physics A: mathematical and general", vol. 14, no. 453, (1981).

[2] L. Kang, W. F. Zhong, Z. G. Lu and F. W. Hong, "Chinese Physics B.”, vol. 22, no. 120-202, (2013).

[3] M. Ohka, K. Beceren, T. Jin, A. Chami, H. B. Yussof and T. Miyaoka, "International Journal of Social Robotics", vol. 4, no. 65, (2012).

[4] Y. Hao, Y. Gong, L. Wang, X. Ma and C. Yang, "Chaos, Solitons \& Fractals", vol. 44, no. 260, (2011).

[5] S. Guo, L. Mei, Y. Li and Y. Sun, "Physics Letters A", vol. 376, no. 407, (2012).

[6] A. S. Hareesh and V. Chandrasekaran, "Pattern Analysis and Applications", vol. 1, (2013).

[7] Z. Wang, J. Gao, Q. Zhou, K. Li and J. Peng, "Journal of Applied Geophysics", vol. 98, no. 176, (2013).

[8] S. Das, I. Pan and S. Das, "ISA transactions", vol. 52, no. 550, (2013).

[9] G. Litak and M. Borowiec, "Nonlinear Dynamics", vol. 1, (2014).

[10] S. Zhong, K. Wei, S. Gao and H. Ma, "Journal of Statistical Physics", vol. 150, no. 867, (2013).

[11] H. G. Tian and L. M. Kang, "Chinese Physics Letters", vol. 29, no. 60204, (2012).

[12] L. Gammaitoni, M. Löcher, A. Bulsara, P. Hänggi, J. Neff, K. Wiesenfeld, W. Ditto and M. E. Inchiosa, "Physical review letters", vol. 82, no. 4574, (1999).

[13] R. Yang and A. Song, "International Journal of Modern Physics B", vol. 26, (2012).

[14] G. Marucci, S. Levantino, P. Maffezzoni and C. Samori, (2013).

[15] Y. Zheng, H. Li, B. Deng and M. Lin, "International Journal of Signal Processing”, Image Processing and Pattern Recognition, vol. 6, no. 275, (2013).

[16] M. Lin and Y. M. Huang, "Journal of Vibration Engineering", vol. 2, no. 5, (2008).

\section{Authors}

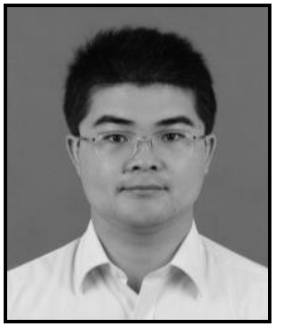

Yongjun Zheng, He is now a Ph.D. candidate of College of Electrical Engineering at Zhejiang University, meanwhile, he is associate professor of College of Metrology and Measurement Engineering at China Jiliang University. His current research interests include different aspects of signal processing, fractional systems and intelligent measurement systems.

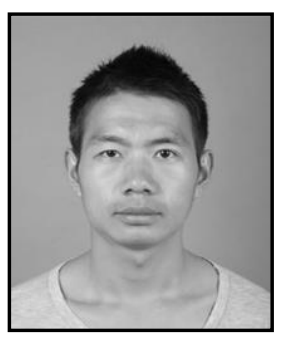

Fei Wang, He is now he is master student of China Jiliang University. His current research interests include different aspects of fractional Systems and intelligent measurement systems. 
International Journal of Control and Automation Vol. 7, No. 11 (2014) 